\title{
Composite odontoma with 23 denticles in a pediatric patient: A case report
}

\author{
D) Paôla Caroline da Silva Mira ${ }^{1}$, (D) Jéssica Silva Peixoto Bem², (1) Andresa Vieira da Silva ${ }^{3}$, \\ (D) Marcio Santos de Carvalho ${ }^{4}$, (1) Marcelo Rodrigues Azenha ${ }^{5}$, (D) Christiano Oliveira-Santos ${ }^{6}$, \\ (D) Maria Bernadete Sasso Stuani ${ }^{7}$, (10) Carolina Paes Torres ${ }^{8}$
}

\section{Highlights}

Odontoma occurs more frequently during the permanent dentition phase in children, adolescents, and young adults.
Even though odontomas are usually not associated with painful symptoms, their presence may trigger major occlusal, phonetic, and aesthetic disorders.
Treatment plan for odontomas should involve a more comprehensive approach considering not only its surgical removal, but also the potential consequences caused by presence of the lesion.
1 Master, Department of Pediatric Clinics, University of São Paulo (USP), Brazil

2 Master student, Department of Pediatric Clinics, University of São Paulo (USP), Brazil

3 Specialization student, Department of Pediatric Clinics, University of São Paulo (USP), Brazil

${ }^{4}$ Master student, Department of Pediatric Clinics, University of São Paulo (USP), Brazil

5 Professor, Department of Stomatology, Public Health, and Forensic Dentistry, University of São Paulo (USP), Brazil

${ }^{6}$ Associate, Professor, Department of Stomatology, Public Health, and Forensic Dentistry, University of São Paulo (USP), Brazil

7 Associate, Professor, Department of Pediatric Clinics, University of São Paulo (USP), Brazil

8 Professor, Department of Pediatric Clinics, University of São Paulo (USP), Brazil

\section{Correspondence:}

Department of Pediatric Clinics, School of Dentistry of Ribeirão Preto, University of São Paulo (USP) - Ribeirão Preto/SP, Brazil E-mail address: paola.mira@usp.br

Received: 02 March 2021

Accepted: 15 April 2021

Online First: 21 April 2021

\section{Abstract}

Compound odontoma is an odontogenic tumor of ectomesenchymal origin containing tooth-like structures. Occasionally, this lesion may trigger occlusal, phonetic, and aesthetic disorders. The aim is to report a case of a compound odontoma containing 23 denticles in the anterior region of the maxilla of an infant patient. A 12-year-old male patient was referred for orthodontic treatment at Ribeirão Preto School of Dentistry, University of São Paulo (Ribeirão Preto, Brazil) complaining of a large diastema between the upper central incisors. Panoramic radiograph revealed the presence of a radiopaque lesion with tooth-like structures surrounded by a radiolucent rim in the anterior region of the maxilla, associated with distal displacement of the left central incisor. Surgical approach under general anesthesia revealed and extracted 23 denticles. Post-operative Cone beam computed tomography (CBCT) confirmed removal of all denticles, and further orthodontic treatment could be planned. Multidisciplinary involvement of pediatric dentist, oral and maxillofacial surgeon, and orthodontist was essential to achieve full functional, aesthetic, and psychosocial success.

Keywords: Odontoma; Pediatric Dentistry; Surgery 


\section{INTRODUCTION}

Odontoma is an odontogenic tumor of ectomesenchymal origin containing anomalous tooth-like structures called denticles (i.e. compound odontoma), or characterized by a mass of irregular hard tissues (i.e. complex odontoma). $\cdot^{1,2}$

Although idiopathic, the formation of an odontoma may be associated with a history of trauma and local infections. Odontoma occurs more frequently during the permanent dentition phase in children, adolescents, and young adults. The compound type tends to affect the anterior region of the maxilla, with a prevalence that varies between 9 to $37 \%$, while the complex type is more common in the posterior region of the maxilla and mandible, with prevalence ranging from 5 to $30 \% .^{3}$

Even though odontomas are usually not associated with painful symptoms, their presence may trigger major occlusal, phonetic, and aesthetic disorders. The delay in the eruption of a permanent tooth in the anterior region of the maxilla is generally the chief complaint that leads to the radiographic investigation and subsequent diagnosis of the anomaly. 4,5

Once diagnosed, the treatment plan for odontomas should involve a more comprehensive approach considering not only their surgical removal, but also the potential consequences caused by their presence. When a compound odontoma is associated with failure of the eruptive process of a permanent tooth, it is essential to establish a strategy including surgical approach and corrective orthodontic treatment to normalize occlusion, phonetics, and aesthetics, to minimize functional alterations, psychological effects, and to strengthen the patient's selfesteem. Therefore, the aim is to report a clinical case of a compound odontoma containing 23 denticles in the anterior region of the maxilla of an infant patient, emphasizing and discussing the surgical approach.

\section{CASE REPORT}

A 12-year-old male patient was referred for orthodontic treatment at Ribeirão Preto School of Dentistry, University of São Paulo (Ribeirão Preto, Brazil) complaining of a large diastema between the upper central incisors. Written informed consent was signed by his legal guardian during his first visit.

Upon clinical examination, the patient had overall good oral hygiene, and did not present active carious lesions or periodontal disease. However, in addition to the interincisal diastema, it was observed that the left lateral incisor was positioned palatally, behind the upper left central incisor (Figure 1). Medical history was unremarkable.

Panoramic radiograph revealed the presence of a heterogeneous radiopaque lesion surrounded by a radiolucent rim in the anterior region of the maxilla, close to the midline, associated with distal displacement of the left central incisor (Figure 1). The internal aspect of the lesion consisted of multiple tooth-like structures, with radiographic appearances of enamel, dentin and pulp, indicating the presence of multiple denticles. The typical radiographic presentation led to the diagnostic hypothesis of a compound odontoma.

The patient was referred to the Pediatric Dentistry clinic and the treatment plan was established in collaboration with an oral and maxillofacial surgeon. Considering the location of the lesion close to the nasal cavity, potential stress to the patient, and his history of anxiety and fear of dental treatment, a surgical approach under general anesthesia in a hospital environment was proposed. 


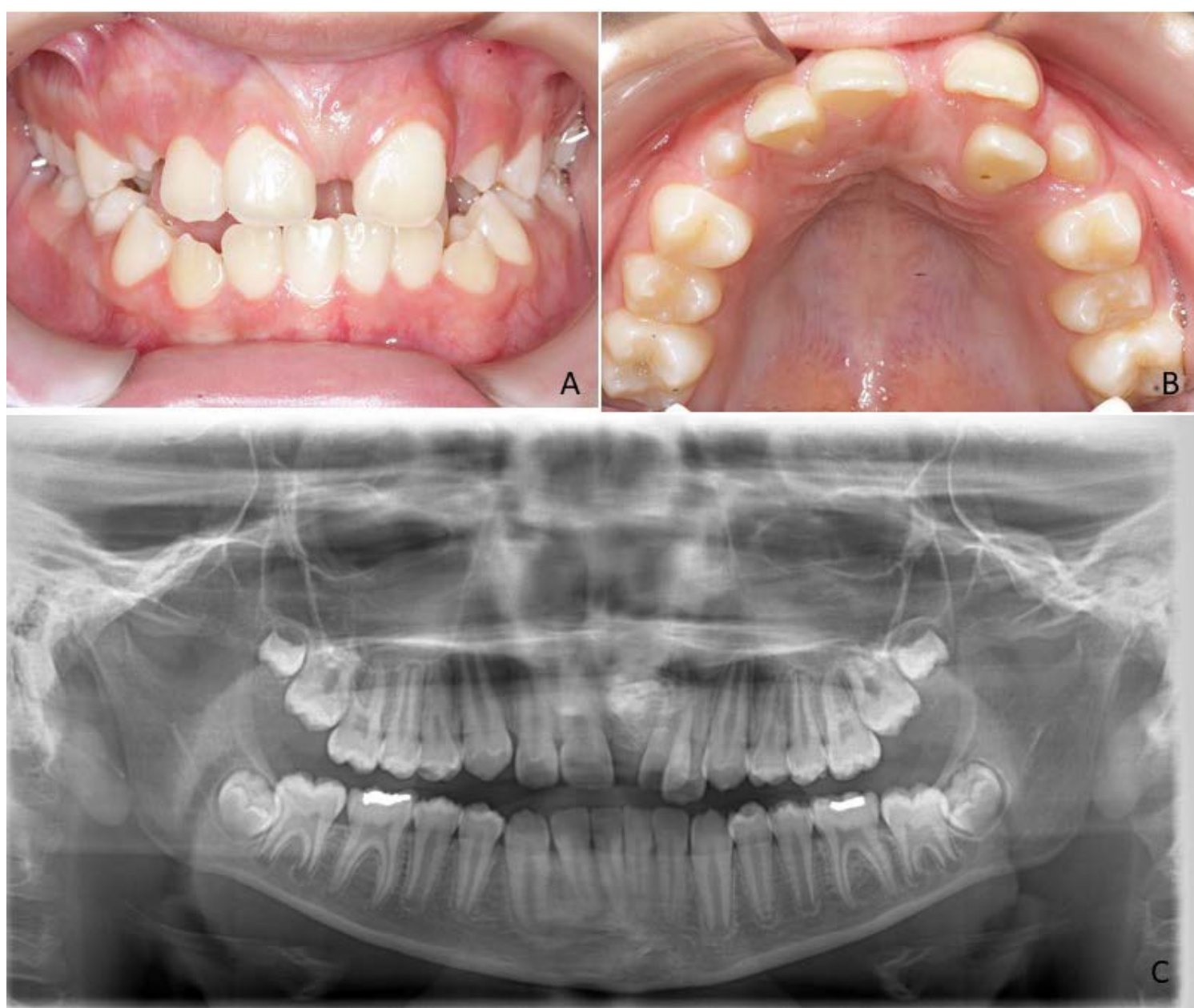

Figure 1. A and B: Front intraoral photograph. C: Initial panoramic radiograph. Radiopaque mass visible between right and left maxillary incisor

Patient underwent medical and pre-anesthetic evaluations, in which no changes were found that would contraindicate the surgical procedure under general anesthesia. Preoperative eight-hour fasting was recommended.

After deep sedation of the patient and antisepsis of the operative field, the surgical procedure started with the buccal access achieved by two parallel parapapillary incisions, from the attached gingiva over the upper central incisors towards the vestibule, for flap detachment and access to the lesion site, followed by its extraction (Figure 2). For this procedure, osteotomy using a manual instrument was satisfactory, due to the small width of the bone in the region.

After the access to the lesion, four denticles were removed using surgical elevators. Initial transoperative judgement and revision of the panoramic radiograph suggested that all the denticles had been removed. However, further surgical exploration revealed the presence of other tooth-like structures and a total of 23 denticles were extracted (Figure 2). Final diagnosis of compound odontoma was established.

Subsequent to irrigation with $0.9 \%$ saline (Equiplex ®, Pharmaceutical Industry, GO, Brazil), aspiration, and careful examination of the surgical site, homeostasis was performed by compressing sterile gauze in the region and repositioning the flap with simple interrupted suture (Figure 2) with absorbable suture thread (Vicryl®, Ethicon Inc., Somerville, NJ, U.S.A.). At the end of surgery, general anesthesia was reversed. The patient was under observation for a few hours, being discharged later on the same day. 

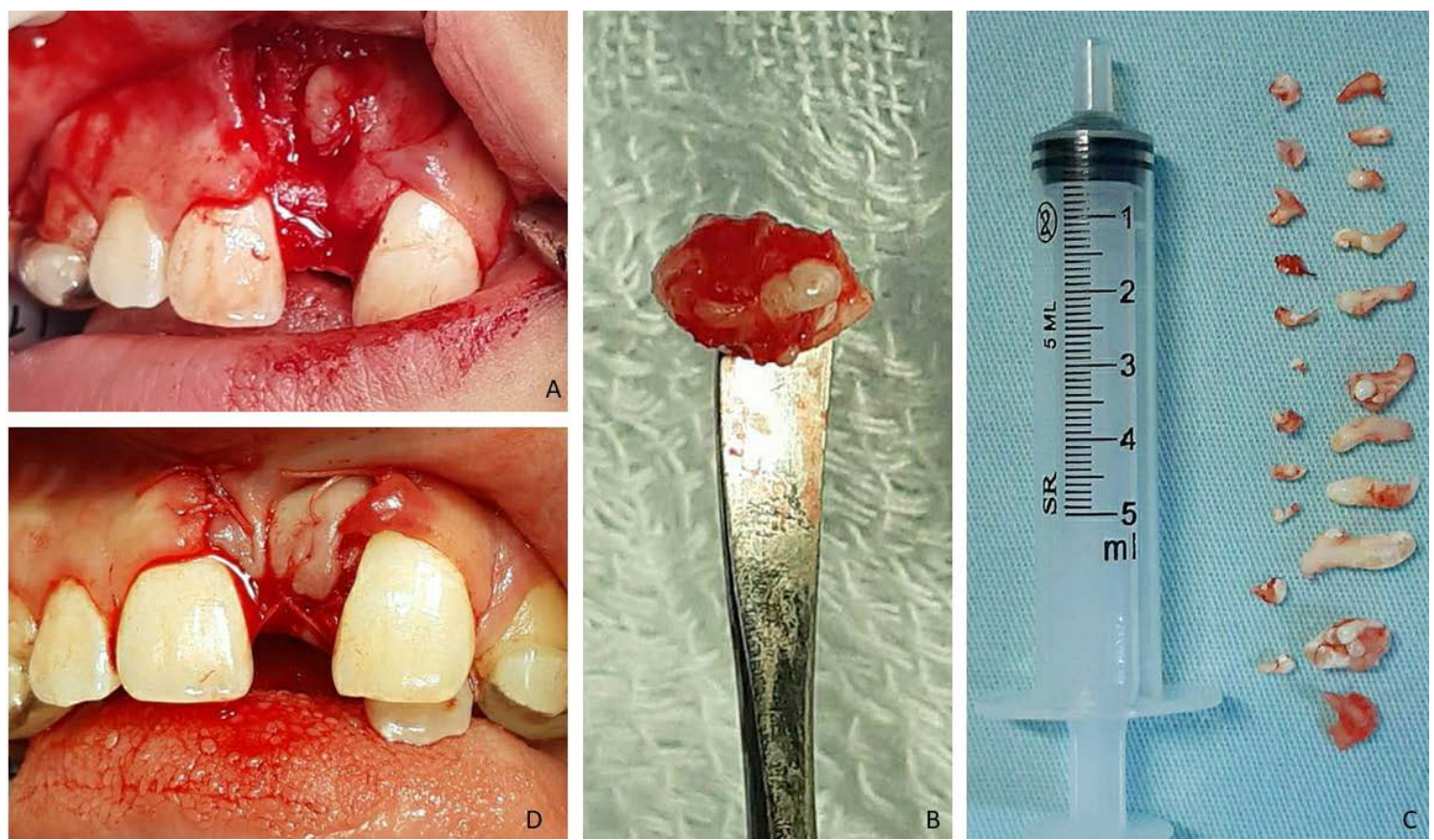

Figure 2. A: Surgical access: Flap opening and bone exposure. B: First mass of odontoma removed, in which the presence of 4 joined denticles can be observed. C: Collection of all fragments removed at the end of the surgery. D:

Completion of surgery with suture in simple points of the surgical wound

One week after surgery, the first postoperative follow-up was performed, indicating normal healing process, absence of spontaneous painful symptoms, edema, or bleeding. Due to the high number of denticles found and to confirm that all fragments had been removed, a CBCT of the region was requested. The removal of all constituents of the compound odontoma was confirmed by the CBCT, without damage to the surrounding structures (Figure 3). The patient currently remains under follow-up, and orthodontic treatment will start after the restrictions imposed by the coronavirus pandemic, which will involve the traction of the displaced lateral incisor and normalization of the occlusion using fixed orthodontic appliances.

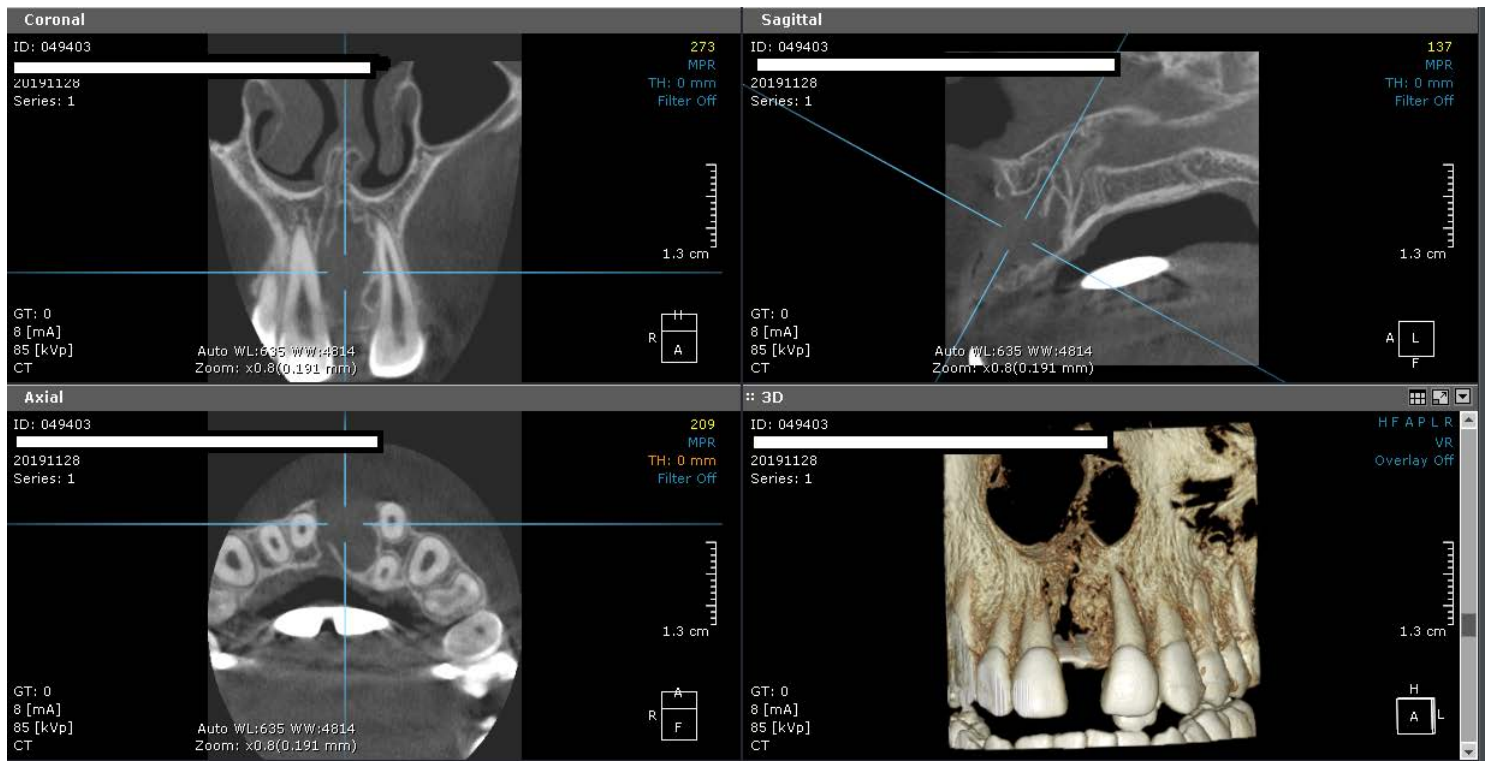

Figure 3. Post-operatory CBCT exam confirms the absence of lesion. Coronal, sagittal and axial planes, in addition to 3D reproduction 


\section{DISCUSSION}

Imaging methods are relevant supplementary exams for diagnosis and treatment planning in Dentistry. Although radiographic examinations have been used for many years in Pediatric Dentistry, the use of three-dimensional imaging has gained more attention in more recent years with the use of CBCT in Dentistry, with its advantages related to elimination of problems related to image overlap in two-dimensional exams. However, its radiation dose is higher than conventional methods, therefore, its use should be performed according to well-established prescription criteria, particularly among pediatric patients. ${ }^{6,7}$ CBCT is indicated for diagnosis and treatment planning of supernumerary teeth, as well as for surgical-orthodontic approaches of impacted teeth. ${ }^{6-9}$

Invasive surgical interventions can be hindered by non-collaborative treatment during the clinical approach due to the dental anxiety presented by the patient according to his psychological maturity, social conditions, and emotional state. ${ }^{10}$ Furthermore, although several non-pharmacological techniques for adapting behavior are available ${ }^{11}$, the approach under general anesthesia was chosen. This option is justified because the diagnosis establish by the team allowed some predictability about the invasive nature of the intervention, and the need to minimize the risk of interference regarding patient's psycho-emotional reactions. Due to the location of the lesion, an appropriate hospital environment would be convenient in case of any complications. Moreover, conscious sedation through inhalation of nitrous oxide/oxygen would not be indicated since it could hinder access to the surgical site.

The 23 denticles removed during the complete excision of the compound odontoma represented, in fact, 19 fragments beyond the number anticipated at the beginning of the surgery. The initial imaging examination brought information from a perspective in two dimensions and did not allow accurate measurement of the anomaly's extent in depth. Thus, adequate planning and diagnostic methods are crucial for an approach with greater predictability and security. 8,12

The surgical plan was made using the panoramic radiography in order to reduce the patient's exposure to ionizing radiation. Despite that, a CBCT examination would have offered valuable information on the extent of the odontoma, and the choice for intervention under general anesthesia for this case would be even further validated.

General anesthesia promoted a smoother and overall faster procedure in addition to the monitorization of the patient's vital signs during the procedure. Also, as it consisted of a localized and short duration procedure, it provided quick recovery and absence of psychological trauma.

The multi-professional treatment planning was extremely important for the therapeutic success in this case. It could be verified by the lesion-free postoperative computed tomography associated with the uneventful healing process (Figure 3). The importance of multidisciplinary involvement is known to be relevant for success of complex treatments in Pediatric Dentistry. ${ }^{1,13}$ In this case, the involvement of the pediatric dentist and the oral and maxillofacial surgeon was essential. Subsequently, the orthodontist could carry out the following stage of the treatment, which would not be possible without the removal of the odontoma.

The presence of interincisal diastemas in children needs further investigation once it might represent a clinical sign of some alterations as dental agenesis, supernumerary teeth (mesiodens), or odontomas. Mesiodens are the most common dental anomaly related to number of teeth found in the anterior region. They are supernumerary teeth in the midline region, and their most common consequence is the delay or impaction of the permanent central incisor. ${ }^{13,14}$ However, compound odontomas should be investigated whenever there are clinical signs suggestive of this 
alteration, such as bulging and delayed eruption of anterior teeth.1,15,16 Imaging is essential for establishing the diagnosis, and CBCT is the method of choice for adequate planning and, consequently, better prognosis. ${ }^{7-9}$

After surgery, corrective orthodontic intervention started to be studied and planned for the proper positioning of the upper incisors and satisfactory results of the occlusal and aesthetic conditions. The importance of adequate imaging exam is highlighted due to its contribution for better observation of diagnostic features of the lesion, treatment plan, and higher surgical predictability. Comprehensive planning involving professionals from different specialties is emphasized, as well as subsequent postoperative follow-up and orthodontic treatment, to maintain patient care until the global resolution of the lesion, aiming at full functional, aesthetic and psychosocial success.

\section{REFERENCES}

1. Altay MA, Ozgur B, Cehreli ZC. Management of a compound odontoma in the primary dentition. J Dent Child 2016;83:98-101

2. Philipsen HP, Reichart PA, Praetorius F. Mixed odontogenic tumours and odontomas. Considerations on interrelationship. Review of the literature and presentation of 134 new cases of odontomas. Oral Oncol 1997;33:86-99

3. de Oliveira BH, Campos V, Marçal S. Compound odontoma-diagnosis and treatment: three case reports. Pediatr Dent 2001;23:151-157

4. Sánchez OH, Berrocal MIL, González JMM. Metaanalysis of the epidemiology and clinical manifestations of odontomas. Med Oral Patol Oral Cir Bucal 2008;13:730-734

5. Janev EJ, Redzep E, Kanurkova L, Tosevska S. Compound odontoma in childhood population (Case Report). J. Morphol Sci 2020;3:73-78

6. Horner K, Islam M, Flygare L, Tsiklakis K, Whaites E. Basic principles for use of dental cone beam computed tomography: consensus guidelines of the European Academy of Dental and Maxillofacial Radiology. Dentomaxillofac Radiol 2020;38:187-195
7. Aps J. Radiography in pediatric dental practice. Clin Dent Rev 2020;4:5-6

8. Katheria BC, Kau CH, Tate R, Chen JW, English J, Bouquot J. Effectiveness of impacted and supernumerary tooth diagnosis from traditional radiography versus cone beam computed tomography. Pediatr Dent 2010;32:304-309

9. Mehta V, Ahmad N. Cone beamed computed tomography in pediatric dentistry: Concepts revisited. J Oral Biol Craniofac Res 2020;10:210211

10. American Academy of Pediatric Dentistry. Management considerations for oral surgery and oral pathology. Pediatr Dent 2017;39:361-370

11. Townsend JA, Wells MH. Behavior guidance of the pediatric dental patient. Pediatr Dent 2019;41:352-370

12. Raupp S, Kramer PF, de Oliveira HW, da Rosa FM, Faraco Junior IM. Application of computed tomography for supernumerary teeth location in pediatric dentistry. Int J Clin Pediatr Dent 2008;32:273-276

13. Monteiro PM, Mira PCS, Arnez MFM, Silva FWGP, Stuani MBS. Mesiodens and maxillary central incisors impaction: multidisciplinary approach to reestablish esthetics and function. Rev Clín Ortod Dent Press 2019;18:120-129

14. Hong J, Lee DG, Park K. Retrospective analysis of the factors influencing mesiodentes eruption. Int J Paediatr Dent 2009;19:343-348

15. Shulman ER, Corio RL. Delayed eruption associated with an odontoma. ASDC J Dent Child 1987;54:205-207

16. Oliver RG, Hodges CG. Delayed eruption of a maxillary central incisor associated with an odontome: report of case. ASDC J Dent Child 1988;55:368-371 


\section{How to cite this article:}

Paôla Caroline da Silva Mira, Jéssica Silva Peixoto Bem, Andresa Vieira da Silva, Marcio Santos de Carvalho, Marcelo Rodrigues Azenha, Christiano Oliveira-Santos, Maria Bernadete Sasso Stuani, Carolina Paes Torres. Composite odontoma with 23 denticles in a pediatric patient: A case report. Contemp Pediatr Dent 2021:2(1):50-56

\section{Declarations}

Acknowledgements: Not applicable.

Conflict of Interest Statement: The authors disclose no potential conflicts of interest.

Ethics Statement: Procedure was explained to the legal guardian and written consent was obtained for the treatment and publication of the case report.

Informed Consent: Written informed consent was signed by his legal guardian during his first visit.

Author contributions: Conception and design: All Authors; Acquisition of data: COS, MBSS, CPT; Interpretation of data: PCSM, SSK; Drafting article: PCSM; Revision artice: JPB, AVS, MSC, MRA; Final approval: All Authors

Funding: This work is not finantiated.

Data Availability: The data used to support the findings of this study can be made available upon request to the corresponding author.

Peer-review: Externally double-blinded peer-reviewed. 